\title{
Parenchyma-sparing hepatectomy (PSH) versus non-PSH for bilobar liver metastases of colorectal cancer
}

\author{
Lidewij Spelt, Daniel Ansari, Max Swanling, Peter Holka, Roland Andersson \\ Clinical Sciences Lund, Skåne University Hospital Lund and Lund University, Sweden
}

\section{Abstract}

\section{Introduction}

Within three years after the diagnosis of colorectal cancer (CRC), 29\% of patients will have developed liver metastases [1]. If these can be resected, 5-year survival of up to $60 \%$ is achievable [2-4]. Curative liver resection is feasible when the metastases can be radically resected whilst sufficient liver volume can be spared [5]. The number of patients eligible

Department of Surgery, Clinical Sciences Lund, Skåne University Hospital Lund and Lund University, Sweden

\section{Conflict of Interest: None}

Correspondence to: Lidewij Spelt, MD, PhD, Department of Surgery, Clinical Sciences Lund Skåne University Hospital Lund and Lund University, SE-221 85 Lund, Sweden,

e-mail: lidewij.spelt@med.lu.se

Received 30 May 2017; accepted 26 September 2017; published online 26 October 2017

DOI: https://doi.org/10.20524/aog.2017.0205 for resection has expanded over time, through advances in surgical procedures and the development of multimodal treatment strategies, in which preoperative chemotherapy is used to decrease tumor burden, and portal vein embolization (PVE) is applied to increase the liver volume spared after resection $[6,7]$.

This has also increased the possibilities for resection in the case of bilobar liver metastases. However, bilobar disease inevitably implies balancing between resecting enough liver tissue to achieve radicality and sparing enough parenchyma to prevent postoperative liver failure. When treating bilobar metastases, resection in practice often implies at least a hemihepatectomy, sacrificing one lobe entirely and sparing very little liver tissue. Previous research has shown that parenchymasparing surgery for CRC liver metastases can be feasible and does not compromise the outcome when compared to more extensive surgery; indeed, it may even have better results in terms of complications and overall survival [8-10]. However, in these studies most patients had only a few metastases and often merely unilobar spread. Another study, including only patients with at least four metastases and thus often with bilobar 
spread, found no certain difference in survival based on the extent of resection [11]. The strategy of parenchyma-sparing hepatectomy (PSH) is presumably most relevant in patients with bilobar disease, where it can be hard to preserve a sufficient amount of liver parenchyma. Yet, studies show that advances are being made in the application of parenchyma-sparing liver surgery, even in patients with technically challenging tumor locations, in which intraoperative ultrasound guidance plays an important role $[12,13]$. Few studies have shown comparable oncological results when applying PSH compared to more extensive surgery (non-PSH) in patients with bilobar disease; whilst PSH might improve perioperative morbidity and mortality [14,15], a true consensus is lacking.

The aim of this retrospective study was to compare the perioperative course and overall survival after liver resection for bilobar CRC metastases in patients who underwent PSH, i.e., segmentectomies and minimal resections in both liver lobes, preserving liver parenchyma, and those who were treated with non-PSH, i.e., an extended hemihepatectomy or hemihepatectomy plus any resection needed on the other lobe.

\section{Patients and methods}

\section{Patients}

All cases of liver resection for bilobar CRC metastases at the Department of Surgery, Lund University Hospital, Sweden, between January 2006 and December 2014, were included in this retrospective cohort study. Patients were excluded if they had undergone previous liver resections or if tumors were deliberately left in situ after operation. The study population was divided into one group treated with PSH and another treated with non-PSH.

Baseline characteristics were collected, including age, sex, comorbidity, American Society of Anesthesiologists (ASA) score, TNM staging of the primary colorectal tumor, and the number and greatest size of the liver metastases. In addition, the performance of preoperative PVE and the administration of chemotherapy were noted.

\section{Treatment}

All patients in both groups underwent liver resection with curative intent. One group was treated with PSH, e.g., multiple wedge resections, atypical resections or segmentectomies on both lobes, the resection entailing in total less than four whole segments. The patients in the other group were subjected to non-PSH, defined as an extended hemihepatectomy or hemihepatectomy plus any resection needed on the other lobe.

\section{Outcome measures}

Outcome measures were intraoperative bleeding, operation time, postoperative hospital stay, radicality of resection and complications. The occurrence of severe complications was noted, implying grade III or higher as defined by the DindoClavien classification system [16]. In addition, the postoperative 30 - and 90-day mortality was calculated, together with the median postoperative overall survival.

\section{Data collection}

Through identification of operation codes, all patients who underwent liver resection for colorectal cancer liver metastases at the Department of Surgery, Lund University Hospital between January 2006 and December 2014, were listed. Their medical files were obtained, after which medical charts, chemotherapy files, operation records and hospital course were carefully reviewed in order to identify the selected patients and obtain all the necessary data. For long-term follow up on survival, the regional administration system was consulted for data on December $6^{\text {th }} 2016$.

The study was approved by the regional human ethics committee at Lund University.

\section{Statistical analysis}

Statistical analysis was performed using SPSS software (IBM SPSS Statistics for Windows, Version 23.0, IBM Corp., Armonk, NY, USA). Comparisons were made using Pearson's chi-square test, Fisher's exact test and the Mann-Whitney $U$ test. Two-sided P-values were used and P-values of $<0.05$ were considered to represent statistical significance. Predictive value for survival was analysed by logistic regression. Unadjusted overall survival analysis was performed by Cox regression and the Kaplan-Meier estimator, using the log-rank test for comparisons between groups. Overall survival adjusting for other covariates was performed using a Cox regression proportional hazards model. Factors taken into account were age, sex, the variables in which the groups differed at baseline, as well as perioperative factors previously shown to be predictors of overall survival, including the occurrence of severe complications and radicality of resection [17-19].

\section{Results}

\section{Patients}

A total of 119 patients with liver resection for bilobar CRC metastases were included. Of these, 59 underwent PSH and 60 patients underwent non-PSH. Both groups had a comparable distribution over time for operation, with 2012 and 2011 as the median years of operation in the PSH group and the non-SPH group, respectively. Patients undergoing $\mathrm{PSH}$ had a higher median age $(\mathrm{P}<0.01)$, but the two groups were comparable regarding sex, ASA score and 
staging of their CRC (Table 1). Although the median size of the largest metastasis was comparable between groups, the median number of metastases was significantly higher in the group with non-PSH and this group also included patients who underwent preoperative PVE, whereas the group with $\mathrm{PSH}$ did not. Preoperative chemotherapy administration was similar in both groups, and most often entailed oxaliplatin-based treatment, followed by irinotecan-based treatment.

\section{Perioperative outcome}

The patients treated with PSH had statistically significantly less median intraoperative bleeding, a shorter operation time and a shorter postoperative stay in hospital (Table 2). Although the division among the different complication grades differed slightly between groups, with more patients having grade 0 or I after PSH and grade II being more common after non-PSH, the occurrence of severe complications was comparable. Radical resection was achieved in similar numbers in both groups.
Within the entire study population there were no perioperative deaths, yielding a total 30-day and 90-day mortality rate of $0 \%$.

\section{Long-term outcome}

The median follow-up time was 35 months among survivors. The median overall unadjusted survival, calculated by the Kaplan-Meier method, was 39 months after PSH and 51 months after non-PSH $(\mathrm{P}=0.139)$. The Kaplan-Meier curve for overall survival in the two groups is presented in Fig. 1, which also shows the unadjusted overall survival using Cox regression $(\mathrm{P}=0.141)$.

Adjusted overall survival was calculated considering age, sex, the patient variables with significant differences or tendencies to differ between groups (ASA score, synchronous versus metachronous metastases, presence of lung metastases, number of liver metastases, size of the largest metastasis and preoperative treatment with PVE), as well as perioperative parameters including the occurrence of severe complications

Table 1 Baseline characteristics in patients undergoing PSH or non-PSH for bilobar CRC metastases

\begin{tabular}{|c|c|c|c|c|}
\hline Characteristic & PSH n=59 (IQR or \%) & Non-PSH $\mathrm{n}=60$ (IQR or \%) & $\mathrm{P}$ & Missing \\
\hline Median age in years & $69(63-76)$ & $65(61-69)$ & $<0.01$ & $0 \%$ \\
\hline Sex, male : female & $35: 24(59.3 \%: 40.7 \%)$ & $39: 21(65 \%: 35 \%)$ & 0.573 & $0 \%$ \\
\hline Year of liver resection & $2012(2010-2013)$ & $2011(2008-2013)$ & 0.121 & $0 \%$ \\
\hline \multicolumn{5}{|l|}{ ASA-score } \\
\hline I & $2(3.4 \%)$ & $7(11.7 \%)$ & 0.092 & $0 \%$ \\
\hline II & $35(59.3 \%)$ & $39(65 \%)$ & & \\
\hline III & $22(37.3 \%)$ & $14(23.3 \%)$ & & \\
\hline \multicolumn{5}{|l|}{ T-stage of CRC } \\
\hline 1 & $1(1.9 \%)$ & $0(0 \%)$ & 0.759 & $16.8 \%$ \\
\hline 2 & $3(5.8 \%)$ & $4(8.5 \%)$ & & \\
\hline 3 & $32(61.5 \%)$ & $29(61.7 \%)$ & & \\
\hline 4 & $16(30.8 \%)$ & $14(29.8 \%)$ & & \\
\hline \multicolumn{5}{|l|}{ N-stage of CRC } \\
\hline 0 & $13(24.5 \%)$ & $17(33.3 \%)$ & 0.243 & $12.6 \%$ \\
\hline 1 & $24(45.3 \%)$ & $15(29.4 \%)$ & & \\
\hline 2 & $16(30.2 \%)$ & $19(37.3 \%)$ & & \\
\hline Synchronous metastases & $29(51.8 \%)$ & $38(64.4 \%)$ & 0.190 & $3.4 \%$ \\
\hline Lung metastases & $10(17.9 \%)$ & $2(3.4 \%)$ & $<0.05$ & $4.2 \%$ \\
\hline Median preoperative CEA-level in $\mu \mathrm{g} / \mathrm{L}$ & $5(3-17)$ & $5(3-18)$ & 0.824 & $34.5 \%$ \\
\hline Median number of liver metastases & $2(2-4)$ & $3(2-5)$ & $<0.01$ & $2.5 \%$ \\
\hline Median size of largest metastasis in $\mathrm{mm}$ & $22(15-30)$ & $26.5(17-40)$ & 0.080 & $3.4 \%$ \\
\hline Preoperative chemotherapy & $40(67.8 \%)$ & $47(78.3 \%)$ & 0.220 & $0 \%$ \\
\hline Preoperative PVE & $0(0 \%)$ & $12(20.7 \%)$ & $<0.001$ & $1.7 \%$ \\
\hline Simultaneous RFA & $10(16.9 \%)$ & $10(16.7 \%)$ & 1.000 & $0 \%$ \\
\hline
\end{tabular}

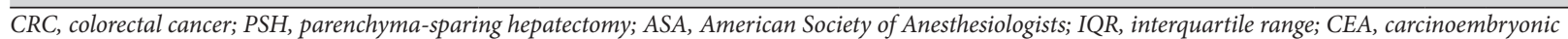
antigen; PVE, portal vein embolization; RFA, radiofrequency ablation 

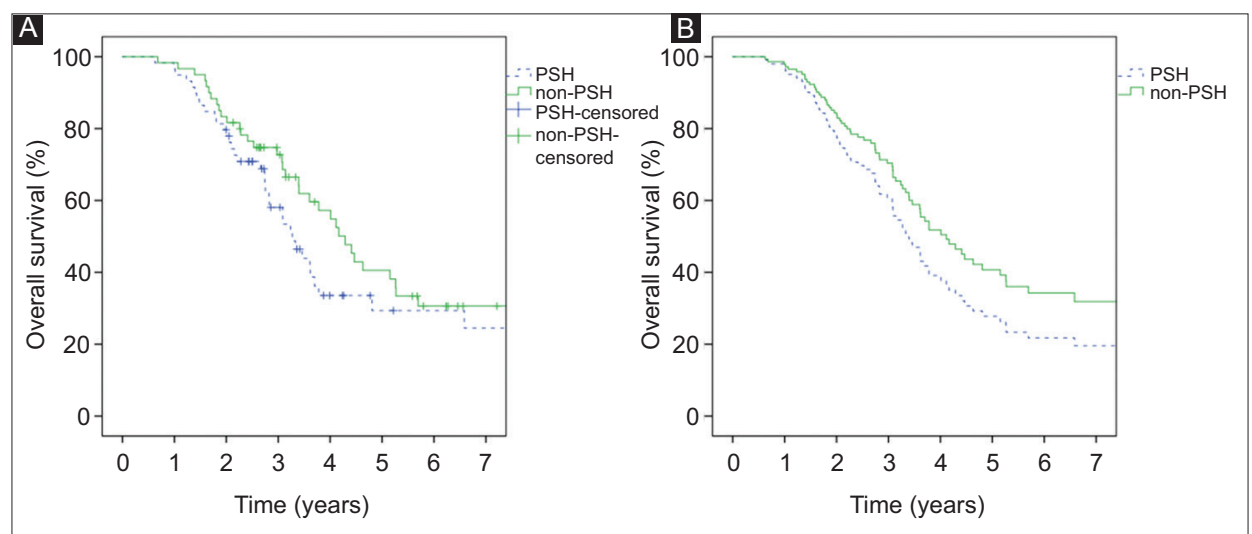

Figure 1 Unadjusted overall survival curves showing overall survival in patients undergoing parenchyma-sparing hepatectomy (PSH) or non-PSH for bilobar colorectal cancer metastases, as demonstrated by the Kaplan-Meier method (A) and unadjusted Cox regression (B)

Table 2 Perioperative outcome in patients undergoing PSH or non-PSH for bilobar CRC metastases

\begin{tabular}{|c|c|c|c|}
\hline Perioperative outcome & PSH $n=59($ IQR or $\%)$ & Non-PSH n=60 (IQR or \%) & $\mathrm{P}$ \\
\hline Median bleeding in $\mathrm{mL}$ & $250(200-500)$ & $600(400-925)$ & $<0.001$ \\
\hline Median operation time in hours & $5 \mathrm{~h}(3.75-7)$ & $7 \mathrm{~h} 25(6-8.75)$ & $<0.001$ \\
\hline Median postoperative stay in days & $7(7-8)$ & $8.5(7-12.5)$ & $<0.01$ \\
\hline Severe complications & $4(6.8 \%)$ & $11(18.3 \%)$ & 0.095 \\
\hline \multicolumn{4}{|l|}{ Complication grade } \\
\hline 0 & $21(35.6 \%)$ & $14(23.3 \%)$ & 0.032 \\
\hline I & $18(30.5 \%)$ & $9(15.0 \%)$ & \\
\hline II & $16(27.1 \%)$ & $26(43.3 \%)$ & \\
\hline III & $3(5.1 \%)$ & $10(16.7 \%)$ & \\
\hline IV & $1(1.7 \%)$ & $1(1.7 \%)$ & \\
\hline Radical resection & $52(88.2 \%)$ & $48(80 \%)$ & 0.317 \\
\hline
\end{tabular}

CRC, colorectal cancer; $P S H$, parenchyma-sparing hepatectomy; IQR, interquartile range

and radicality of resection. Using Cox proportional hazards regression analysis, no statistically significant differences in survival were found when comparing patients who underwent PSH with those who underwent non-PSH (Fig. 2). Hazard ratios for extensive versus limited resection are displayed in Table 3.

\section{Discussion}

The present study is one of few reports focusing on the influence of the extent of liver surgery on perioperative outcome and long-term survival, specifically in patients with bilobar liver metastases. An important argument against performing PSH when dealing with bilobar CRC liver metastases has been the suggestion that this might have a negative impact on radicality. Radicality, i.e., a tumor-free resection margin, is a prognostic factor for long-term survival $[18,20]$, and some have found a higher rate of positive margins when comparing wedge resections to larger anatomical resections [21]. In the current study, however, no difference in radicality was found between
Table 3 Cox multivariable regression analysis for overall survival

\begin{tabular}{lccc}
\hline Overall survival & HR & $\begin{array}{c}95 \% \text { CI for } \\
\text { HR }\end{array}$ & $\mathrm{P}$ \\
\hline $\begin{array}{l}\text { PSH vs. non-PSH, } \\
\text { unadjusted }\end{array}$ & 1.426 & $0.889-2.289$ & 0.141 \\
$\begin{array}{l}\text { PSH vs. non-PSH, adjusted } \\
\text { for covariates }\end{array}$ & 1.375 & $0.669-2.826$ & 0.386 \\
\hline
\end{tabular}

${ }^{a}$ Adjusted for sex, age, ASA-score, synchronous metastases, lung metastases, number of liver metastases, size of the largest metastasis, PVE treatment, radicality of resection and the occurrence of severe complications

$H R$, hazard ratio; CI, confidence interval; $P S H$, parenchyma-sparing hepatectomy; ASA, American Society of Anesthesiologists

groups. This finding is supported by the study of Memeo et al, who also compared patients with bilobar disease undergoing $\mathrm{PSH}$ to those undergoing non-PSH and found similar rates of radical resection [15].

The present study demonstrated differences in perioperative course when comparing both types of surgery. When the resected liver volume was limited, this was associated with a 


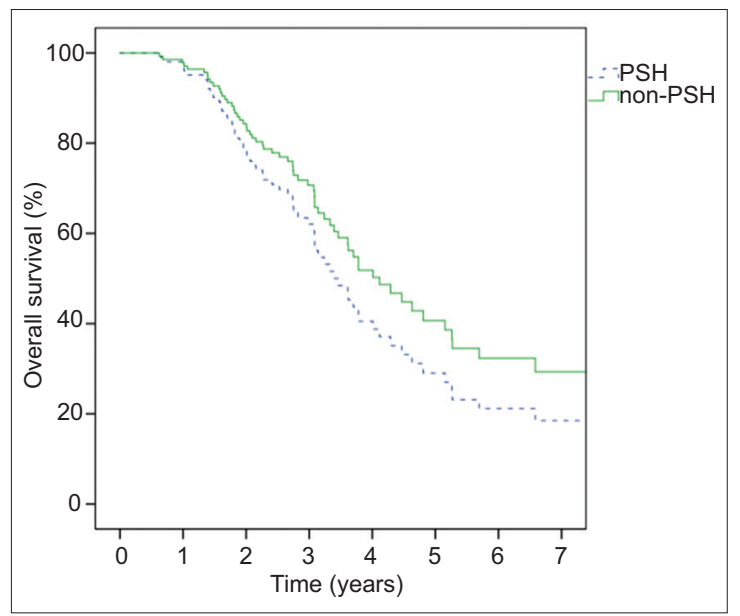

Figure 2 Adjusted overall survival curves showing overall survival in patients undergoing parenchyma-sparing hepatectomy (PSH) or non-PSH for bilobar colorectal cancer metastases, calculated through adjusted Cox regression

smaller amount of perioperative bleeding, a shorter operation time and shorter hospital stay. Also, the division amongst the different grades of complications was different. However, when the number of severe complications was considered, the groups were comparable. A favorable perioperative course after PSH was also reported by Memeo et al, showing fewer complications, although hospital stay was comparable in their study [15]. In the study by Gold et al [14], groups were separated according to different time periods and this showed that less extensive resections were performed during later periods, and were correlated with a decrease in blood loss, hospital stay and 90-day mortality over time. They also showed that extensive resections were related to a higher morbidity.

Besides the possibly favorable perioperative course, another reason to opt for PSH is the possibility of future resections in case the patient suffers from hepatic recurrence. If achievable in these cases, reresection has been shown to be beneficial [22,23], but is more likely to be ruled out in patients with a previous non-PSH and thus less remaining liver tissue.

This study showed comparable overall survival in patients treated with PSH and those who underwent non-PSH. However, one difficulty in the current study, which was a retrospective study with all its limitations, was the difference between the two study groups regarding certain aspects that may have affected outcomes. First of all, the median age was higher in the group with $\mathrm{PSH}$, reported to negatively influence survival [2]. Also, this group included 10 patients who had lung metastases at the time of liver resection, whilst the group with non-PSH only contained two patients with lung metastases. Since the overall 3-year survival for patients with lung metastases from CRC is reported to be only in the range of $11-14 \%$ [24], the presence of lung metastases would thus be considered as a negative prognostic factor. Additionally, the median number of liver metastases differed between the two groups. Several studies found a higher number of liver metastases to be related to worse survival [25-27], whereas others have not judged this to be a negative predictive factor [28]. A higher number of liver

\section{Summary Box}

\section{What is already known:}

- The number of patients who are considered for treatment with liver resection for colorectal cancer (CRC) metastases is increasing, partly due to advances in multimodal treatment strategies that allow for larger resections to be performed

- A balance needs to be found between resecting enough liver tissue to achieve radicality and sparing enough parenchyma to prevent postoperative liver failure

- Especially in the case of bilobar liver metastases this can be a challenge, and few studies have started to compare parenchyma-sparing operation techniques with more extensive resections such as (extended) hemihepatectomies

\section{What the new findings are:}

- Outcomes in patients treated with parenchymasparing hepatectomy (PSH) were compared to outcomes in those treated with non-PSH, showing no difference in radicality

- The amount of intraoperative bleeding was smaller and the median operation time and hospital stay were shorter after PSH compared to non-PSH, while overall survival was comparable

- This study supports the use of PSH for bilobar CRC liver metastases, when possible

metastases might also have influenced the surgeon to consider non-PSH in the first place, and the possible prognostic value of this parameter should be taken into account. To adjust for the abovementioned factors and also taking other marginally differing variables into account, an adjusted survival analysis was performed with Cox regression analysis and this still showed no statistically significant difference in survival between the two groups. This finding is in accordance with previous studies involving patients with bilobar liver metastases, also showing comparable overall survival in patients with extensive and with limited surgery $[14,15]$. Therefore, the presumption that PSH might negatively influence long-term outcome is contradicted.

In conclusion, when comparing the perioperative outcome and long-term survival in CRC patients who underwent PSH or non-PSH for bilobar liver metastases, our study could not demonstrate any disadvantage when using PSH. Instead, possible advantages compared to non-PSH-e.g., less intraoperative bleeding, shorter operation time and shorter hospital stay-were noted. Furthermore, minimizing the liver resection did not appear to influence radicality. Although extensive resections often are unavoidable because of tumor location and technical aspects, our findings imply that the 
application of PSH is safe when feasible, thereby sparing liver parenchyma and facilitating possible future liver resections for local recurrences.

\section{References}

1. Leporrier J, Maurel J, Chiche L, Bara S, Segol P, Launoy G. A population-based study of the incidence, management and prognosis of hepatic metastases from colorectal cancer. Br J Surg 2006;93:465-474.

2. Wei AC, Greig PD, Grant D, Taylor B, Langer B, Gallinger S. Survival after hepatic resection for colorectal metastases: a 10-year experience. Ann Surg Oncol 2006;13:668-676.

3. Tan MC, Castaldo ET, Gao F, et al. A prognostic system applicable to patients with resectable liver metastasis from colorectal carcinoma staged by positron emission tomography with $[18 \mathrm{~F}]$ fluoro-2-deoxy-D-glucose: role of primary tumor variables. $J$ Am Coll Surg 2008;206:857-868.

4. Pawlik TM, Scoggins CR, Zorzi D, et al. Effect of surgical margin status on survival and site of recurrence after hepatic resection for colorectal metastases. Ann Surg 2005;241:715-722, discussion 722-724.

5. Pawlik TM, Schulick RD, Choti MA. Expanding criteria for resectability of colorectal liver metastases. Oncologist 2008; 13:51-64.

6. Folprecht G, Gruenberger T, Bechstein WO, et al. Tumour response and secondary resectability of colorectal liver metastases following neoadjuvant chemotherapy with cetuximab: the CELIM randomised phase 2 trial. Lancet Oncol 2010;11:38-47.

7. Azoulay D, Castaing D, Smail A, et al. Resection of nonresectable liver metastases from colorectal cancer after percutaneous portal vein embolization. Ann Surg 2000;231:480-486.

8. von Heesen M, Schuld J, Sperling J, et al. Parenchyma-preserving hepatic resection for colorectal liver metastases. Langenbecks Arch Surg 2012;397:383-395.

9. Stewart GD, O’Súilleabháin $\mathrm{CB}$, Madhavan KK, Wigmore SJ, Parks RW, Garden OJ. The extent of resection influences outcome following hepatectomy for colorectal liver metastases. Eur J Surg Oncol 2004;30:370-376.

10. Karanjia ND, Lordan JT, Quiney N, Fawcett WJ, Worthington TR, Remington J. A comparison of right and extended right hepatectomy with all other hepatic resections for colorectal liver metastases: a ten-year study. Eur J Surg Oncol 2009;35:65-70.

11. Tanaka K, Shimada H, Matsumoto C, et al. Impact of the degree of liver resection on survival for patients with multiple liver metastases from colorectal cancer. World J Surg 2008;32:2057-2069.

12. Torzilli G, Vigano L, Gatti A, et al. Twelve-year experience of "radical but conservative" liver surgery for colorectal metastases: impact on surgical practice and oncologic efficacy. HPB (Oxford) 2017;19:775-784.

13. Torzilli G, Montorsi M, Donadon M, et al. "Radical but conservative" is the main goal for ultrasonography-guided liver resection: prospective validation of this approach. J Am Coll Surg 2005;201:517-528.
14. Gold JS, Are C, Kornprat P, et al. Increased use of parenchymalsparing surgery for bilateral liver metastases from colorectal cancer is associated with improved mortality without change in oncologic outcome: trends in treatment over time in 440 patients. Ann Surg 2008;247:109-117.

15. Memeo R, de Blasi V, Adam R, et al. Parenchymal-sparing hepatectomies (PSH) for bilobar colorectal liver metastases are associated with a lower morbidity and similar oncological results: a propensity score matching analysis. HPB (Oxford) 2016;18:781-790.

16. Dindo D, Demartines N, Clavien PA. Classification of surgical complications: a new proposal with evaluation in a cohort of 6336 patients and results of a survey. Ann Surg 2004;240:205-213.

17. Mavros MN, de Jong M, Dogeas E, Hyder O, Pawlik TM. Impact of complications on long-term survival after resection of colorectal liver metastases. Br J Surg 2013;100:711-718.

18. Tranchart H, Chirica M, Faron M, et al. Prognostic impact of positive surgical margins after resection of colorectal cancer liver metastases: reappraisal in the era of modern chemotherapy. World J Surg 2013;37:2647-2654.

19. Sadot E, Groot Koerkamp B, Leal JN, et al. Resection margin and survival in 2368 patients undergoing hepatic resection for metastatic colorectal cancer: surgical technique or biologic surrogate? Ann Surg 2015;262:476-485.

20. Are C, Gonen M, Zazzali K, et al. The impact of margins on outcome after hepatic resection for colorectal metastasis. Ann Surg 2007;246:295-300.

21. DeMatteo RP, Palese C, Jarnagin WR, Sun RL, Blumgart LH, Fong Y. Anatomic segmental hepatic resection is superior to wedge resection as an oncologic operation for colorectal liver metastases. J Gastrointest Surg 2000;4:178-184.

22. Jönsson K, Gröndahl G, Salö M, Tingstedt B, Andersson R. Repeated liver resection for colorectal liver metastases: a comparison with primary liver resections concerning perioperative and long-term outcome. Gastroenterol Res Pract 2012;2012:568214.

23. Kulik U, Bektas H, Klempnauer J, Lehner F. Repeat liver resection for colorectal metastases. Br J Surg 2013;100:926-932.

24. Mitry E, Guiu B, Cosconea S, Jooste V, Faivre J, Bouvier AM. Epidemiology, management and prognosis of colorectal cancer with lung metastases: a 30-year population-based study. Gut 2010;59:1383-1388.

25. Angelsen JH, Viste A, Løes IM, et al. Predictive factors for time to recurrence, treatment and post-recurrence survival in patients with initially resected colorectal liver metastases. World J Surg Oncol 2015;13:328.

26. Gomez D, Zaitoun AM, De Rosa A, et al. Critical review of the prognostic significance of pathological variables in patients undergoing resection for colorectal liver metastases. HPB (Oxford) 2014;16:836-844.

27. Malik HZ, Hamady ZZ, Adair R, et al. Prognostic influence of multiple hepatic metastases from colorectal cancer. Eur J Surg Oncol 2007;33:468-473.

28. Minagawa M, Makuuchi M, Torzilli G, et al. Extension of the frontiers of surgical indications in the treatment of liver metastases from colorectal cancer: long-term results. Ann Surg 2000;231:487-499. 